\title{
Ed Brinksma
}

\section{Kim Guldstrand Larsen (Eds.)}

Computer Aided Verification

14th International Conference, CAV 2002

Copenhagen, Denmark, July 2002

Proceedings 
Lecture Notes in Computer Science

2404

Edited by G. Goos, J. Hartmanis, and J. van Leeuwen 


\section{Springer}

\section{Berlin}

Heidelberg

New York

Barcelona

Hong Kong

London

Milan

Paris

Tokyo 
Ed Brinksma Kim Guldstrand Larsen (Eds.)

\section{Computer Aided Verification}

14th International Conference, CAV 2002

Copenhagen, Denmark, July 27-31, 2002

Proceedings 


\section{Series Editors}

Gerhard Goos, Karlsruhe University, Germany

Juris Hartmanis, Cornell University, NY, USA

Jan van Leeuwen, Utrecht University, The Netherlands

Volume Editors

Ed Brinksma

University of Twente, Department of Computer Science

P. O. Box 217, 7500 AE Enschede, The Netherlands

E-mail: brinksma@cs.utwente.nl

Kim Guldstrand Larsen

Aalborg University, Department of Computer Science

Fredrik Bajers Vej 7, 9220, Aalborg Ø, Denmark

E-mail:kgl@cs.auc.dk

Cataloging-in-Publication Data applied for

Die Deutsche Bibliothek - CIP-Einheitsaufnahme

Computer aided verification : 14th international conference ; proceedings /

CAV 2002, Copenhagen, Denmark, July 27 - 31, 2002. Ed Brinksma ;

Kim Guldstrand Larsen (ed.). - Berlin ; Heidelberg ; New York ; Barcelona ;

Hong Kong ; London ; Milan ; Paris ; Tokyo : Springer, 2002

(Lecture notes in computer science ; Vol. 2404)

ISBN 3-540-43997-8

CR Subject Classification (1998): F.3, D.2.4, D.2.2, F.4.1, I.2.3, B.7.2, C.3

ISSN 0302-9743

ISBN 3-540-43997-8 Springer-Verlag Berlin Heidelberg New York

This work is subject to copyright. All rights are reserved, whether the whole or part of the material is concerned, specifically the rights of translation, reprinting, re-use of illustrations, recitation, broadcasting, reproduction on microfilms or in any other way, and storage in data banks. Duplication of this publication or parts thereof is permitted only under the provisions of the German Copyright Law of September 9, 1965, in its current version, and permission for use must always be obtained from Springer-Verlag. Violations are liable for prosecution under the German Copyright Law.

Springer-Verlag Berlin Heidelberg New York

a member of BertelsmannSpringer Science+Business Media GmbH

http://www.springer.de

(C) Springer-Verlag Berlin Heidelberg 2002

Printed in Germany

Typesetting: Camera-ready by author, data conversion by DA-TeX Gerd Blumenstein

Printed on acid-free paper $\quad$ SPIN $10873641 \quad 06 / 3142 \quad 543210$ 


\section{Preface}

This volume contains the proceedings of the conference on Computer Aided Verification (CAV 2002), held in Copenhagen, Denmark on July 27-31, 2002. CAV 2002 was the 14 th in a series of conferences dedicated to the advancement of the theory and practice of computer-assisted formal analysis methods for software and hardware systems. The conference covers the spectrum from theoretical results to concrete applications, with an emphasis on practical verification tools, including algorithms and techniques needed for their implementation. The conference has traditionally drawn contributions from researchers as well as practitioners in both academia and industry.

This year we received 94 regular paper submissions out of which 35 were selected. Each submission received an average of 4 referee reviews. In addition, the CAV program contained 11 tool presentations selected from 16 submissions. For each tool presentation, a demo was given at the conference. The large number of tool submissions and presentations testifies to the liveliness of the field and its applied flavor.

The CAV 2002 program included a tutorial day with three invited tutorials by Wolfgang Thomas (Aachen) on Infinite Games and Verification, Patrick Cousot (ENS Paris) on Abstraction in Software Verification and Thomas A. Henzinger (Berkeley) on The Symbolic Approach to Hybrid Systems. The conference also included two invited talks by Sharad Malik (Princeton) on The Quest for Efficient Boolean Satisfiability Solvers and Gerard J. Holzmann (Bell Labs) on Software Analysis and Model Checking. In addition, there were three workshops associated with CAV 2002:

- PAPM-PROBMIV: Process Algebras and Performance Modeling/Probabilistic Methods in Verification.

- RT-TOOLS: Workshop on Real-Time Tools.

- RV: Run-Time Verification.

The publication of these workshop proceedings was managed by their respective chairs, independently of the present proceedings.

We would like to thank all the Program Committee members and the subreferees who assisted in their work. Our thanks also go to the Steering Committee members and last year's organizers for their helpful advice. The Local Organization Chair, Jens Christian Godskesen, deserves our gratitude for his contributions throughout the preparations. We would also like to thank the invited speakers and invited tutorial speakers, the authors of submitted papers, and all the participants of the conference. Special thanks go to Brian Nielsen for installing and managing the START Conference system and to Ole Høgh Jensen for the production of the final proceedings.

This year, CAV was part of the Federated Logic Conference (FLoC 2002), and was organized jointly with CADE (Conference on Automated Deduction), 
FME (Formal Methods Europe), ICL (International Conference on Logic Programming), LICS (Logic in Computer Science), RTA (Rewriting Techniques and Applications), and TABLEAUX (Automated Reasoning with Analytic Tableaux and Related Methods). In particular, the invited talk given by Sharad Malik was joint with CADE 2002, and the paper also appears, in identical form, in the proceedings of CADE 2002. In addition, FLoC included 31 workshops associated with the different conferences. We would like to acknowledge the help of the FLoC 2002 steering committee Moshe Y. Vardi (General Chair), Neil D. Jones (Conference Chair), Ulrich Firbach (CADE), Edmund M. Clarke (CAV), Dines Bjørner (CAV), Catuscia Palamidessi (ICLP), Samson Abramsky (LICS), Nachum Dershowitz (RTA), Reiner Hähnle (TABLEAUX), Harald Ganzinger (Associate General Chair), and Dana Scott (IFCOLOG).

Finally, we gratefully acknowledge support from IBM, Esterel Technologies, IT-U of Copenhagen, the Department of Computer Science at Aalborg University, Twente University, and BRICS.

May 2002

Ed Brinksma and Kim Guldstrand Larsen 


\section{Program Committee}

Thomas Ball (Microsoft Research)

David Basin (Freiburg)

Armin Biere (ETH Zürich)

Ed Brinksma (Twente, co-chair)

Werner Damm (Oldenburg)

E. Allen Emerson (Austin)

Alain Finkel (Cachan)

Nicolas Halbwachs (IMAG Grenoble)

John Hatcliff (Kansas State)

Klaus Havelund (NASA)

Thomas A. Henzinger (Berkeley)

Andreas Kuehlmann (Cadence)

Orna Kupferman (Jerusalem)
Kim G. Larsen (Aalborg, co-chair)

Tim Leonard (Compaq)

Ken McMillan (Cadence)

Kedar Namjoshi (Bell Labs)

Doron A. Peled (Austin)

Amir Pnueli (Weizmann Inst.)

Natarajan Shankar (SRI)

Joseph Sifakis (IMAG Grenoble)

Fabio Somenzi (Boulder)

Bernhard Steffen (Dortmund)

Yaron Wolfsthal (IBM)

Wang Yi (Uppsala)

\section{Steering Committee}

Edmund M. Clarke (CMU)

Amir Pnueli (Weizmann Inst.)

Robert Kurshan (Cadence)

Joseph Sifakis (IMAG Grenoble)

\section{Local Organizer}

Jens Christian Godskesen (IT-U of Copenhagen)

\section{Sponsors}

IBM

Esterel Technologies

IT-U of Copenhagen
Dept. of CS, Aalborg University

Twente University

BRICS

\section{Referees}

Parosh Abdulla

Nina Amla

Pablo Argon

Tamarah Arons

Cyrille Artho

Evgueni Asarin

Gadiel Auerbach

Abdelwaheb Ayari

Kamel Barkaoui

Sharon Barner
Gerd Behrmann

Shoham Ben-David

Johan Bengtsson

Sergey Berezin

Roderick Bloem

Bernard Boigelot

Michele Boreale

Ahmed Bouajjani

Patricia Bouyer

Marius Bozga
Ionut Buricea

Franck Cassez

Pavol Cerny

Hana Chockler

Thomas Colcombet

Dennis Dams

Alexandre David

Xiaoqun Du

M. Duflot

Bruno Dutertre 
Matthew Dwyer

Niklas Een

Cindy Eisner

Kousha Etessami

Monica Farkash

Jean-Claude Fernandez

D. Fink

Dana Fisman

Emmanuel Fleury

Martin Fränzle

Carl Chr. Frederiksen

L. Fribourg

Dimitra Giannakopoulou

Patrice Godefroid

Jean Goubault-Larrecq

Susanne Graf

Claudia Gsottberger

Elsa Gunter

Alan Hartman

Frederic Herbreteau

Holger Hermanns

Gerard Holzmann

Hardi Hungar

Radu Iosif

S. Iyer

Damir Jamsek

Somesh Jha

Ranjit Jhala

HoonSang Jin

Damien Joly

Bengt Jonsson

Bernhard Josko

Marcin Jurszinski

Vineet Kahlon

M. Kaltenbach

Joost-Pieter Katoen

Felix Klaedtke

Nils Klarlund

Jens Knoop

Olga Kouchnarenko

Hillel Kugler
Robert P. Kurshan

Yassine Lakhnech

Rom Langerak

Jim Larus

Jerome Leroux

Xavier Leroy

Bing Li

Angelika Mader

Monika Maidl

Rupak Majumdar

Oded Maler

Freddy Mang

Panagiotis Manolios

Nicolas Markey

Ken McMillan

Jon Millen

Mark Minas

Sebastian Moedersheim

Oliver Möller

In-Ho Moon

Remi Morin

Laurent Mounier

Leonardo de Moura

Markus Müller-Olm

Uwe Nestmann

Juergen Niehaus

Oliver Niese

Thomas Noll

Abelardo Pardo

Corina Pasareanu

Charles Pecheur

Wojciech Penczek

Paul Pettersson

Claudine Picaronny

Nir Piterman

Shaz Qadeer

Sriram K. Rajamani

Kavita Ravi

S. Ray

E. Reeber

Iris Reuveni
Grigore Rosu

Sitvanit Ruah

Harald Ruess

John Rushby

Oliver Rüthing

Theo Ruys

Hassen Saidi

Jun Sawada

Viktor Schuppan

Helmut Seidl

Ohad Shaham

Elad Shahar

Gil Shurek

Maria Sorea

Robert Staerk

Christian Stehno

M. Sustik

Gregoire Sutre

Ashish Tiwari

Richard J. Trefler

Jan Tretmans

Stavros Tripakis

Tomas Uribe

Moshe Vardi

Miroslav Velev

Luca Vigano

S. Vinod

Willem Visser

T. Wahl

Chao Wang

Farn Wang

Ingo Wegener

Jon Whittle

Thomas Wilke

Harro Wimmel

Burkhart Wolff

Heisung Yoo

Emmanuel Zarpas

Wenhui Zhang

C. Zhou

Lenore Zuck 


\section{Table of Contents}

\section{Invited Talks}

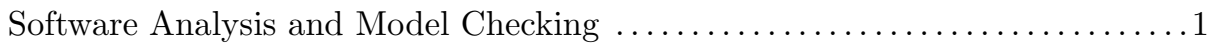
Gerard J. Holzmann

The Quest for Efficient Boolean Satisfiability Solvers ................... 17

Lintao Zhang and Sharad Malik

\section{Invited Tutorials}

On Abstraction in Software Verification ............................. 37

Patrick Cousot and Radhia Cousot

The Symbolic Approach to Hybrid Systems $\ldots \ldots \ldots \ldots \ldots \ldots \ldots \ldots \ldots$

Thomas A. Henzinger

Infinite Games and Verification (Extended Abstract of a Tutorial) ........58 Wolfgang Thomas

\section{Symbolic Model Checking}

Symbolic Localization Reduction

with Reconstruction Layering and Backtracking

Sharon Barner, Daniel Geist, and Anna Gringauze

Modeling and Verifying Systems Using a Logic of Counter Arithmetic

with Lambda Expressions and Uninterpreted Functions $\ldots \ldots \ldots \ldots \ldots \ldots 78$

Randal E. Bryant, Shuvendu K. Lahiri, and Sanjit A. Seshia

Combining Symmetry Reduction and Under-Approximation

for Symbolic Model Checking

Sharon Barner and Orna Grumberg

\section{Abstraction/Refinement and Model Checking}

Liveness with $(0,1, \infty)$-Counter Abstraction $\ldots \ldots \ldots \ldots \ldots \ldots \ldots \ldots \ldots \ldots \ldots \ldots$ Amir Pnueli, Jessie Xu, and Lenore Zuck

Shared Memory Consistency Protocol Verification

Against Weak Memory Models: Refinement via Model-Checking . . ....... 123

Prosenjit Chatterjee, Hemanthkumar Sivaraj, and Ganesh Gopalakrishnan

Automatic Abstraction Using Generalized Model Checking ..............137

Patrice Godefroid and Radha Jagadeesan 


\section{Compositional/Structural Verification}

Property Checking via Structural Analysis

Jason Baumgartner, Andreas Kuehlmann, and Jacob Abraham

Conformance Checking for Models

of Asynchronous Message Passing Software $\ldots . \ldots \ldots \ldots \ldots \ldots \ldots \ldots . \ldots \ldots$

Sriram K. Rajamani and Jakob Rehof

A Modular Checker for Multithreaded Programs ...................... 180

Cormac Flanagan, Shaz Qadeer, and Sanjit A. Seshia

\section{Timing Analysis}

Automatic Derivation of Timing Constraints by Failure Analysis .........195

Tomohiro Yoneda, Tomoya Kitai, and Chris Myers

Deciding Separation Formulas with SAT ......................... 209

Ofer Strichman, Sanjit A. Seshia, and Randal E. Bryant

Probabilistic Verification of Discrete Event Systems

Using Acceptance Sampling ..................................223

Håkan L. S. Younes and Reid G. Simmons

\section{SAT Based Methods}

Checking Satisfiability of First-Order Formulas

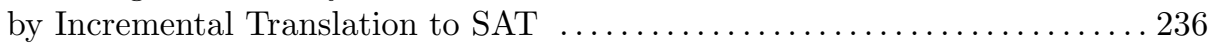

Clark W. Barrett, David L. Dill, and Aaron Stump

Applying SAT Methods in Unbounded Symbolic Model Checking ........ 250 Ken L. McMillan

SAT Based Abstraction-Refinement Using ILP

and Machine Learning Techniques ............................ 265

Edmund Clarke, Anubhav Gupta, James Kukula, and Ofer Strichman

Semi-formal Bounded Model Checking ........................... 280

Jesse D. Bingham and Alan J. Hu

\section{Symbolic Model Checking}

Algorithmic Verification of Invalidation-Based Protocols ............... 295

Marco Bozzano and Giorgio Delzanno

Formal Verification of Complex Out-of-Order Pipelines by Combining

Model-Checking and Theorem-Proving ............................309

Christian Jacobi 
Automated Unbounded Verification of Security Protocols .324

Yannick Chevalier and Laurent Vigneron

\section{Tool Presentations}

Exploiting Behavioral Hierarchy for Efficient Model Checking . . . . . . . . . 338 Rajeev Alur, Michael McDougall, and Zijiang Yang

IF-2.0: A Validation Environment for Component-Based Real-Time Systems

Marius Bozga, Susanne Graf, and Laurent Mounier

The AVISS Security Protocol Analysis Tool .......................... 349 Alessandro Armando, David Basin, Mehdi Bouallagui, Yannick Chevalier, Luca Compagna, Sebastian Mödersheim, Michael Rusinowitch, Mathieu Turuani, Luca Viganò, and Laurent Vigneron

SPeeDI - A Verification Tool for Polygonal Hybrid Systems

Eugene Asarin, Gordon Pace, Gerardo Schneider, and Sergio Yovine

NuSMV 2: An OpenSource Tool for Symbolic Model Checking ...

Alessandro Cimatti, Edmund Clarke, Enrico Giunchiglia,

Fausto Giunchiglia, Marco Pistore, Marco Roveri, Roberto Sebastiani, and Armando Tacchella

The d/dt Tool for Verification of Hybrid Systems

Eugene Asarin, Thao Dang, and Oded Maler

\section{Infinite State Model Checking}

Model Checking Linear Properties of Prefix-Recognizable Systems . . . . . . 371 Orna Kupferman, Nir Piterman, and Moshe Y. Vardi

Using Canonical Representations of Solutions to Speed Up Infinite-State Model Checking Tatiana Rybina and Andrei Voronkov

On Discrete Modeling and Model Checking for Nonlinear Analog Systems

Walter Hartong, Lars Hedrich, and Erich Barke

\section{Compositional/Structural Verification}

Synchronous and Bidirectional Component Interfaces Arindam Chakrabarti, Luca de Alfaro, Thomas A. Henzinger, and Freddy Y. C. Mang 
Interface Compatibility Checking for Software Modules

Arindam Chakrabarti, Luca de Alfaro, Thomas A. Henzinger,

Marcin Jurdziński, and Freddy Y.C. Mang

Practical Methods for Proving Program Termination

Michael A. Colón and Henny B. Sipma

\section{Extended Model Checking}

Evidence-Based Model Checking

Li Tan and Rance Cleaveland

Mixing Forward and Backward Traversals

in Guided-Prioritized BDD-Based Verification

Gianpiero Cabodi, Sergio Nocco, and Stefano Quer

Vacuum Cleaning CTL Formulae

Mitra Purandare and Fabio Somenzi

\section{Tool Presentations}

CVC: A Cooperating Validity Checker

Aaron Stump, Clark W. Barrett, and David L. Dill

$\chi$ Chek: A Multi-valued Model-Checker

Marsha Chechik, Arie Gurfinkel, and Benet Devereux

PathFinder: A Tool for Design Exploration

Shoham Ben-David, Anna Gringauze, Baruch Sterin, and Yaron Wolfsthal

Abstracting $\mathrm{C}$ with abC

Dennis Dams, William Hesse, and Gerard Holzmann

AMC: An Adaptive Model Checker

Alex Groce, Doron Peled, and Mihalis Yannakakis

\section{Code Verification}

Temporal-Safety Proofs for Systems Code

Thomas A. Henzinger, Ranjit Jhala, Rupak Majumdar,

George C. Necula, Grégoire Sutre, and Westley Weimer

\section{Regular Model Checking and Acceleration}

Extrapolating Tree Transformations

Ahmed Bouajjani and Tayssir Touili 
Regular Tree Model Checking

Parosh Aziz Abdulla, Bengt Jonsson, Pritha Mahata, and Julien d'Orso

Compressing Transitions for Model Checking

Robert Kurshan, Vladimir Levin, and Hüsnü Yenigün

\section{Model Reduction}

Canonical Prefixes of Petri Net Unfoldings ........................ 582

Victor Khomenko, Maciej Koutny, and Walter Vogler

State Space Reduction by Proving Confluence

Stefan Blom and Jaco van de Pol

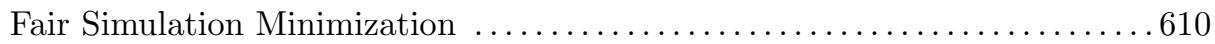

Sankar Gurumurthy, Roderick Bloem, and Fabio Somenzi

Author Index 